\title{
Excel como herramienta docente de las asignaturas de Microeconomía
}

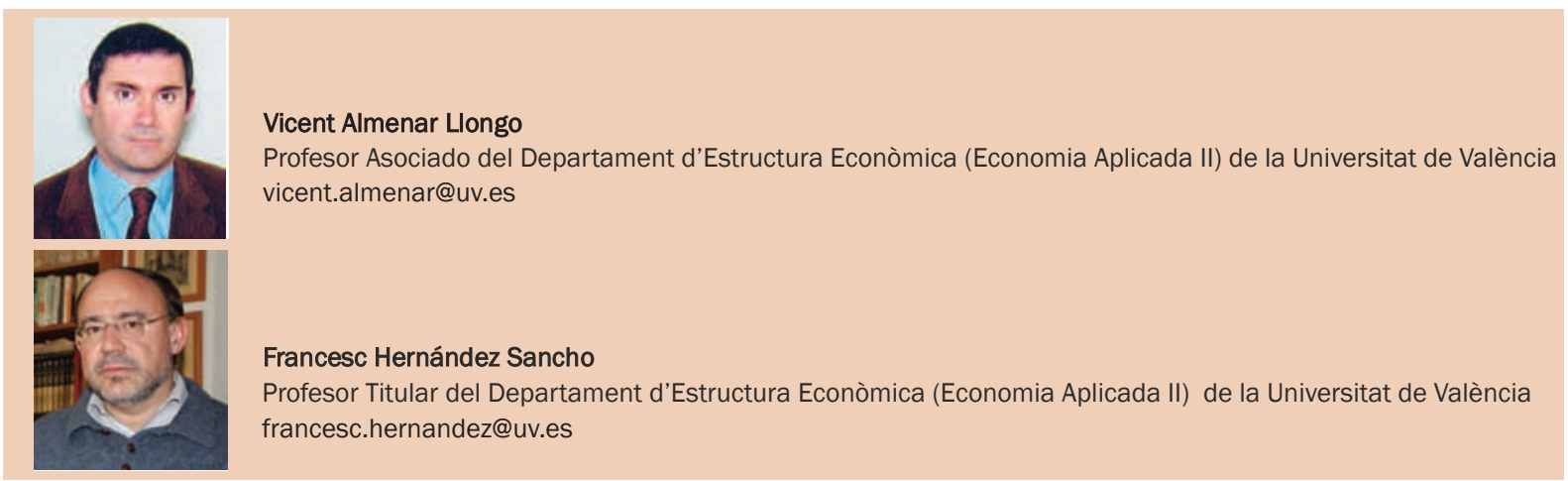

|Fecha presentación: 20/10/2009 | Aceptación: 20/11/2009 |Publicación: 04/12/2009

\begin{abstract}
Resumen
El presente trabajo trata sobre la utilización del programa Excel en la docencia de las asignaturas relacionadas con la Microeconomía. Se trata de especificar la forma algebraica de un modelo microeconómico para mostrar al alumno cómo es posible la resolución del problema utilizando la hoja de cálculo Excel. De esta forma, el alumno adquiere competencias específicas del área de estudio, al tiempo que competencias transversales basadas en habilidades básicas en el manejo del ordenador o las habilidades informáticas básicas.
\end{abstract}

Palabras clave: EEES, innovación, Microeconomía, Excel

\section{Resum}

El present treball tracta sobre la utilització del programa Excel en la docència de les assignatures relacionades amb la Microeconomia. Es tracta d'especificar la forma algebraica d'un model microeconòmic per a mostrar a l'alumne com és possible la resolució del problema utilitzant la fulla de càlcul Excel. D'aquesta manera, l'alumne adquireix competències específiques de l'àrea d'estudi, al temps que competències transversals basades en habilitats bàsiques en l'ús de l'ordinador o les habilitats informàtiques bàsiques.

Paraules clau: EEES, innovació, Microeconomia, Excel

\begin{abstract}
This paper deals with the use of the Excel program in teaching subjects related to Microeconomics. The paper intends to specify algebraically a microeconomic model to show the student the possibility of solving problems by using an Excel spreadsheet. In this way, the student acquires both specific skills in the microeconomics field and core skills based on basic skills in the use of the computer or basic computer skills.
\end{abstract}

Keywords: EHEA, innovation, Microeconomics, Excel 


\section{Introducción}

El presente trabajo trata sobre la utilización del programa Excel en la docencia de las asignaturas relacionadas con la microeconomía (Introducción a la Economía, Microeconomía, Economía Agraria, Economía Mundial/Internacional, etc.), con el objetivo de que el alumno adquiera competencias específicas del área de estudio, al tiempo que adquiere competencias basadas en habilidades básicas en el manejo del ordenador o las habilidades informáticas básicas.

Como es bien conocido, Microsoft Office Excel es una aplicación para manejar hojas de cálculo. Una hoja de cálculo (o programa de hojas de cálculo) es un programa que permite manipular datos numéricos y alfanuméricos dispuestos en forma de tablas (la cual es la unión de filas y columnas) para realizar cálculos automáticos y complejos con fórmulas y funciones de números que están en la tabla, y dibujar distintos tipos de gráficas.

Microsoft Office Excel es una hoja de cálculo que presenta una serie de ventajas para el usuario: es un software disponible en la mayoría de los ordenadores y que esconde una potencialidad no suficientemente conocida por los usuarios; permite la recomputación inteligente de celdas (las celdas que dependen de otra celda que ha sido modificada se actualizan al instante); permite definir la apariencia de las hojas de cálculo (las fuentes, atributos de carácter y apariencia de las celdas); tiene una amplia capacidad gráfica; e incluye (desde 1993) Visual Basic para Aplicaciones (VBA), un lenguaje de programación basado en Visual Basic, que añade la capacidad para automatizar tareas en Excel y para proporcionar las funciones definidas por el usuario (UDF) para su uso en las hojas de trabajo.

Excel no sólo bebe considerarse una herramienta sumamente útil para las clases de estadística, matemáticas y matemáticas financieras (gráficas, fórmulas financieras, presentación de informes...) sino también para las de Economía. De hecho, existe bibliografía sobre el uso de Excel en la docencia en Economía a la que podemos hacer referencia: Barry (1995a), Barry (1995b), Katz y Becker (1999), Whigham y Whyte (1999), Cahill y Kosicki (2000), Tohamy y Mixon, (2000), Mixon y Tohamy (2000), Cahill y Kosicki (2001), Tohamy y Mixon (2002) y Mixon (2005)1.

Pero en la misma práctica, se propone al alumno que resuelva el problema usando programas de álgebra computacional como Maple, TI-Nspire CAS o Mathematica, con el objetivo de que el alumno conozca la solución del problema a la que debe de llegar mediante el uso de la hoja de cálculo
Excel (lo cual debería serle de gran ayuda para las tareas que implica la práctica).

Maple ${ }^{2}$ es un programa matemático capaz de realizar cálculos simbólicos ${ }^{3}$, algebraicos y de álgebra computacional, que comercializa Waterloo Maple Inc. o Maplesoft (www.maplesoft.com) desde 1988. Maple cuenta con algoritmos numéricos para poder estimar resultados y resolver problemas donde soluciones exactas no existan, un gran conjunto de herramientas gráficas que permiten visualizar los resultados (alguna vez complejos) obtenidos, y un lenguaje de programación para que el usuario pueda desarrollar sus propias funciones y programas. Además, el usuario de Maple puede crear hojas de trabajo interactivas basadas en cálculos matemáticos en las que puede cambiar un dato o una ecuación y actualizar todas las soluciones inmediatamente.

TI-Nspire CAS, sucesor del software Derive ${ }^{4}$, es la nueva herramienta de Texas Instruments para la enseñanza y aprendizaje de las Matemáticas. Consta, entre otras características, de un sistema algebraico computacional, un sistema de geometría dinámica, una hoja de cálculo, un módulo sobre representaciones gráficas, un paquete de estadística y manejo de recopilación de datos.

Por último, Mathematica ${ }^{5}$ es un programa desarrollado por Wolfram Research (www.wolfram.com) y utilizado en áreas científicas, de matemáticas, ingeniería y computacionales. Se trata de un sistema de álgebra computacional y de un lenguaje de programación de propósito general. Se divide en dos partes: el kernel o núcleo que desempeña los cálculos, y el front end o interfaz que despliega los resultados y permite al usuario interactuar con el núcleo como si fuera un documento.

Entre las características de Mathematica cabe destacar: la capacidad de solucionar sistemas de ecuaciones (ordinarias, parciales o diferenciales); la disponibilidad de bibliotecas de funciones elementales y especiales para matemáticas; soporte de matrices; herramientas numéricas y simbólicas para cálculo de variable continua o discreta; herramientas de visualización de datos en $2 \mathrm{D}$ y $3 \mathrm{D}$; una colección de bases de datos, incluida información socio-económica; una funcionalidad como procesador técnico de palabras (cuaderno de notas), incluyendo un editor de fórmulas, etc.

Así, nuestra propuesta consiste en la utilización conjunta de Excel y los programas de álgebra computacional en las asignaturas del área de microeconomía, como herramientas para apoyar su aprendizaje y su enseñanza, ya que

\footnotetext{
1 Asimismo, destacamos la existencia de las siguientes webs con recursos disponibles: Problems in Microeconomics http://www.bus.msu.edu/econ/brown/pim/ (Byron W. Brown, 2002); Basic Microeconomics http://www.boisestate.edu/econ//reynol/web/excel index.htm (R. Larry Reynolds, 2007); Economics in Excel http://csob.berry.edu/faculty/economics/ (Mixon y Tohamy, 2008).

${ }^{2}$ Actualmente se encuentra disponible en el mercado Maple 15 (versión actualizada en noviembre de 2009), y para abril de 2010 está prevista la versión Maple 16.

${ }^{3}$ Los programas de cálculo simbólico pueden ser definidos como programas para ordenadores personales (PC) que sirven para trabajar con matemáticas usando las notaciones propias (simbólicas) de esta ciencia. Estos programas son capaces de hacer derivadas, integrales, límites, y muchas otras operaciones matemáticas. Suelen tener capacidades gráficas (representación de curvas y funciones) y capacidades de calculadora científica (sus capacidades numéricas suplen sobradamente a la mejor de las calculadoras).

${ }^{4}$ Derive es un software que Texas Instruments ya no comercializa en sus versiones para PC y lo implementa ahora en su nueva tecnología de calculadoras graficas, las TI-Nspire: en la evolución de Derive a TI-CAS, pasó de ser una aplicación de ordenador a estar incluido en las calculadoras TI-89 y TI-Nspire CAS. Derive, fue concebido como un programa de cálculo simbólico, muy potente y versátil para el cálculo matemático avanzado al tiempo que muy accesible, intuitivo y sencillo, lo que lo hacía idóneo para iniciarse con este tipo de programas. Entre las capacidades del programa: operaciones con vectores, matrices y determinantes; resolución de ecuaciones y de sistemas de ecuaciones; derivadas, integrales (definidas e indefinidas), series, límites, polinomios de Taylor; representación gráfica de funciones de dos variables; operaciones con polinomios y fracciones algebraicas; etc. Además, Derive se suministraba con varios ficheros de funciones para propósitos diversos, como resolver ecuaciones diferenciales, trabajar en álgebra lineal, etc. (http://www.upv.es/derive/general.htm, consultado el 8-9-2009). Conviene señalar que existen trabajos sobre el uso de Derive en la docencia de la economía: Murphy (1995a) y Murphy (1995b).
}

${ }^{5}$ La versión 7, la más reciente, fue liberada el 18 de noviembre de 2008, y se encuentra disponible para una gran variedad de sistemas operativos. 
muchos temas pueden tratarse más eficientemente de esta forma que usando métodos de enseñanza tradicionales. En las clases de microeconomía se plantean muchos problemas que requieren cálculos extensos y laboriosos, que pueden resolverse apretando una tecla cuando se usan estos programas, eliminando el aspecto más tedioso de muchos cálculos matemáticos. Si dejamos a los programas informáticos los aspectos mecánicos y los algoritmos de la resolución de problemas, los estudiantes pueden concentrarse en el significado de los conceptos microeconómicos (en lugar de enseñar habilidades de cálculo).

\section{Propuesta de prácticas}

Las actividades planteadas consisten en especificar funciones de demanda y de oferta de un bien para obtener, utilizando la hoja de cálculo Excel, el equilibrio de mercado, y posteriormente, variando la condición ceteris paribus, provocar desequilibrios en el mercado (exceso de oferta o de demanda) que tiendan a generar nuevos equilibrios (el uso del programa Maple, TI-Nspire CAS o Mathematica se platea como un apoyo a la resolución de los problemas).

Una primera actividad propuesta consiste en plantear un problema estándar de oferta y demanda de un bien ${ }^{6}$. Se proporciona al alumno la forma algebraica de una función de demanda (la cantidad demandada en función del precio del bien, del precio del bien complementario, del precio del bien sustitutivo, de la renta y de los gustos) y de oferta (precio del bien y precio de un input de producción). Como ejemplo, referimos la práctica al vino peleón:

$$
\begin{aligned}
& Q_{v}^{d}=-3 P_{v}-6 P_{g}+7 P_{c}+1.5 R+350 \\
& Q_{v}^{s}=2,4 P_{v}-7 P_{u}+1500
\end{aligned}
$$

Siendo: $\mathrm{Q}_{\mathrm{V}}^{\mathrm{d}}$ cantidad demandada de vino peleón; $\mathrm{P}_{\mathrm{V}}$ precio de vino peleón; $\mathrm{P}_{\mathrm{g}}$ precio de la gaseosa; $\mathrm{P}_{\mathrm{c}}$ precio de la cerveza; $\mathrm{R}$ renta; $\mathrm{Q}_{\mathrm{V}}^{3}$ cantidad ofrecida de vino peleón; $\mathrm{P}_{\mathrm{u}}$ precio de la uva.
La primera parte de la práctica consistiría en calcular a partir de las expresiones de oferta y demanda del mercado del vino peleón, el precio y la cantidad de equilibrio utilizando Maple, TI-Nspire CAS o Mathematica.

La segunda parte de la práctica consistiría la construcción de una hoja de cálculo Excel que permita: calcular el equilibrio de mercado; calcular los valores de equilibrio si se modifica(n) valor(es) de las variables que han sido consideradas como constantes o los parámetros de las ecuaciones de oferta y demanda; representar gráficamente el equilibrio de mercado y los desplazamientos de las curvas de oferta y de demanda. Una posible hoja de cálculo que sirviese como solución sería la que se muestra en la Figura 1.

A partir de este punto, y en función de nuestros objetivos docentes, puede plantearse a los alumnos ampliaciones de la actividad. Por ejemplo: estudiar las características del bien vino peleón (normal o inferior, complementario o sustitutivo) ${ }^{7}$; plantear cuestiones sobre cómo se refleja el cambio en el estado de la tecnología de producción en la oferta, o los cambios en los gustos y la publicidad en la demanda; ampliar la hoja de cálculo realizada introduciendo otras variables que pueden influir sobre las intenciones de demandar (comprar, consumir) u ofertar (vender, producir) vino peleón (por el lado de la demanda, expectativas sobre precios -precios esperados- y variaciones en la población; por el lado de la oferta, introducción de un nuevo input necesario para la producción y de su precio -ejemplo: el factor trabajo y el salario-, variaciones en el número de empresas, impuestos y subvenciones); plantear a los alumnos cuestiones elaboradas a partir de una noticia publicada en prensa con referencia a casos reales, como por ejemplo:

En:

www.elmundo.es/mundodinero/2008/12/29/economi a/1230537279.html se puede acceder a la noticia cuyo titular reza así: "La crisis beneficia las marcas más económicas”. El vino de mesa se impone al reserva. La crisis dispara las ventas de Don Simón un 29\%. Se hunde un 20\% la de los grandes caldos.

Lee este artículo y contesta a la pregunta: ¿consideras

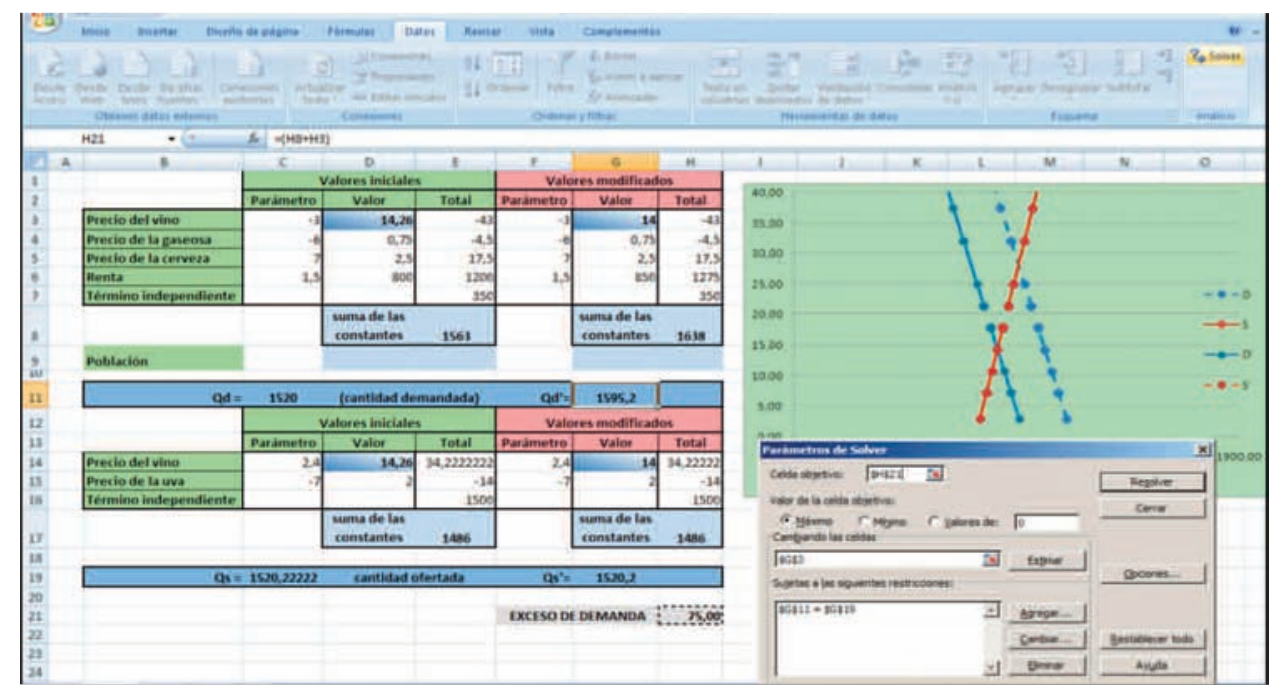

Figura 1

\footnotetext{
${ }^{6}$ Un enfoque tradicional de este problema se puede encontrar en Blanco y Azar (2004), capítulo 2, problema 2.4.

${ }^{7}$ Nuestra experiencia indica que si los alumnos calculan los excesos de oferta y demanda que se producen cada vez que se altera una variable de las que inicialmente se consideraban constantes (junto con la observación de la función de demanda) se facilita la tarea de identificación.
} 


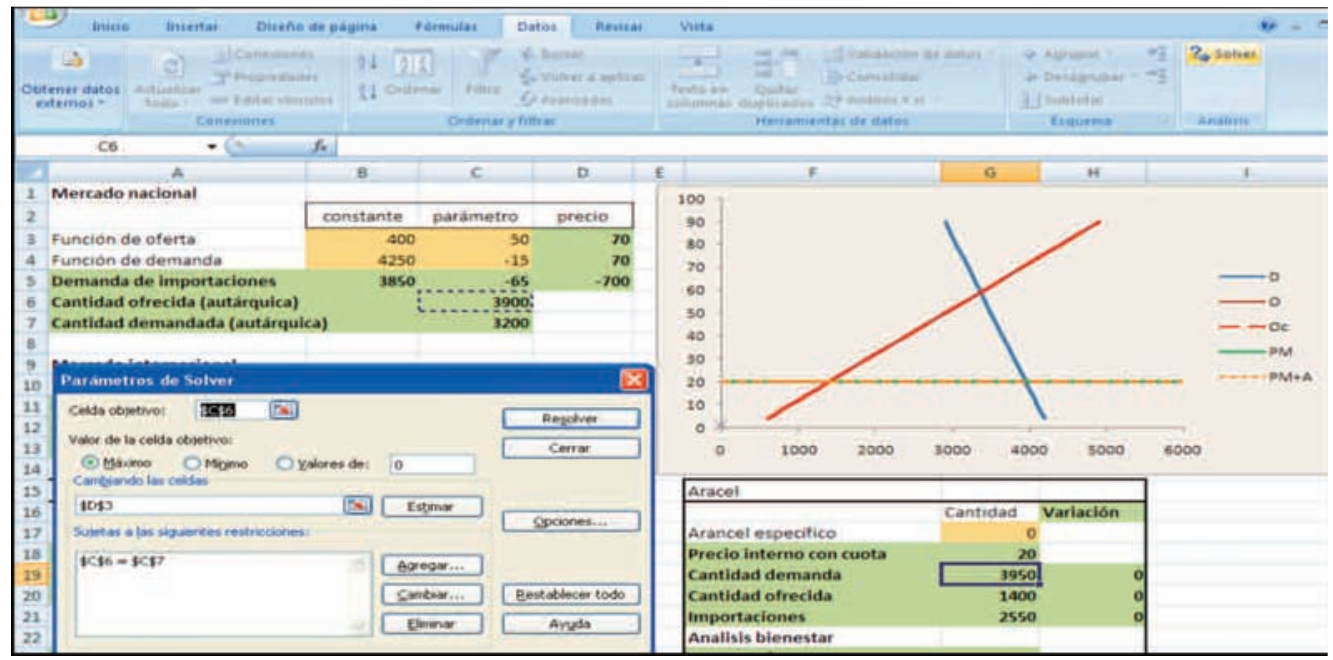

que se han especificado correctamente las formas algebraicas de las funciones de oferta y demanda?

Una segunda actividad propuesta y dirigida a asignaturas de Economía Mundial / Internacional consiste en especificar la forma algebraica de las funciones de demanda y de oferta interna de un bien, y analizar los efectos que tiene el establecimiento de una cuota y de un arancel sobre el comercio de un país pequeño (incluidos los efectos sobre el bienestar de ese país) mediante el uso de la hoja de cálculo Excel. Por ejemplo ${ }^{8}$ :

Sea un país incapaz de afectar a los precios mundiales del vino. Su función de oferta de vino es:

$Q_{v}^{s}=400+50 P_{v}$

y su función de demanda:

$$
Q_{v}^{d}=4250-15 P_{v}
$$

Se trata de contestar a: ¿cómo varía el precio y la cantidad de equilibrio si pasamos de una economía en autarquía a una economía con comercio internacional? ¿qué cambios introduce el establecimiento de una cuota de 1.500 unidades de vino en el precio interno, en la oferta interna y en el bienestar social de la economía?cंqué cambios introduce el establecimiento de un arancel 5 u.m. por unidad importada de vino en el precio interno, en la oferta y la demanda interna, en las importaciones y en el bienestar social de la economía?ċcuál debería ser el valor del arancel para restringir las importaciones al montante de la cuota?

Los pasos a seguir en la práctica deben ser: realizar los cálculos necesarios para la resolución del ejercicio utilizando Maple, TI-Nspire CAS o Mathematica, diseñar en Excel una hoja de cálculo que le permita contestar a todas las pregun- tas del enunciado y representar gráficamente el equilibrio de mercado y los desplazamientos de las curvas de oferta y de demanda. Un ejemplo puede verse en la Figura 2.

Es conveniente referenciar este tipo de prácticas a casos reales que promuevan el debate (individual o en grupos), y un buen medio para ello es plantear a los alumnos cuestiones elaboradas a partir de noticias publicadas en la prensa, por ejemplo:

En http://www.elpais.com.uy/090301/ultmo$401855 /$ ultimomomento/ue-dijo-no-alproteccionismo-unilateral se puede acceder a la noticia cuyo titular reza así: "UE dijo 'no' al proteccionismo unilateral" [...]." Pese a las polémicas de las semanas pasadas, el titular de la Comisión Europea, José Durao Barroso, declaró en la conferencia de prensa final que 'hubo un alto nivel de consenso sobre la necesidad de evitar cualquier tipo de medidas unilaterales proteccionistas dentro de la UE'“.

Leed el artículo de prensa y contestad la pregunta: ¿ipuede considerarse que el proteccionismo sería una solución a la crisis económica actual? Razonad la respuesta.

La tercera práctica propuesta consiste especificar las funciones de demanda y de oferta de un producto agrario, para resaltar las especiales condiciones de los mercados agrarios. Como en las dos prácticas anteriores, el primer paso consiste en especificar la forma algebraica de una función de demanda y de oferta (el precio actual y/o esperado del bien, los precios de los inputs de producción, la superficie deseada (que coincide con la efectivamente plantada) y los rendimientos medios. Como ejemplo se propone el siguiente enunciado:

Supongamos que la producción planeada de chufa de los agricultores de L'Horta de València responde a las variables del siguiente modelo:

${ }^{8}$ Un enfoque tradicional de este problema se puede encontrar en el capítulo 4 Restricciones al comercio internacional del manual de Bengoechea et al. (2002). 

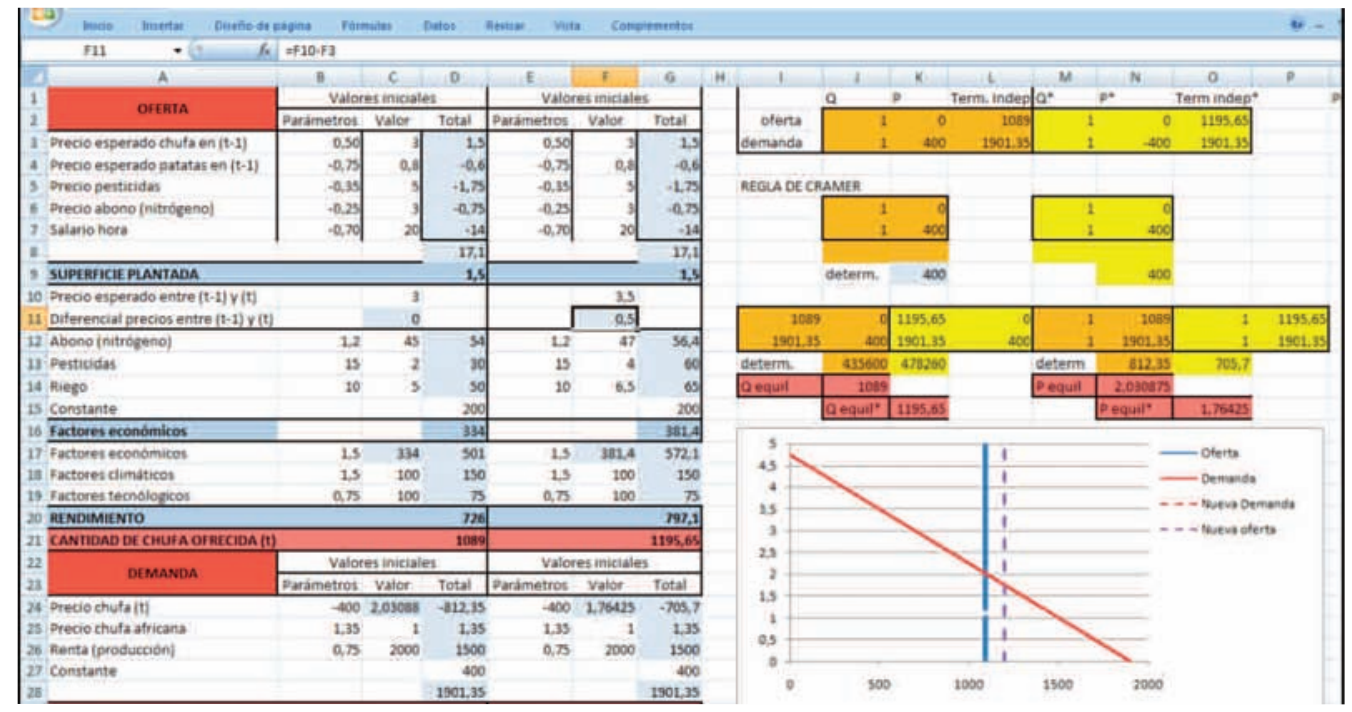

$$
\begin{aligned}
& Q_{x, t}^{s}=R^{e} \cdot S^{e} \\
& S^{e}=\left(17.1+0,5 P_{x, t}^{e}-0.75 P_{P a, s}^{e}-0,35 P_{p e}-0.25 P_{A_{N}}-0.7 w\right) \\
& R^{e}=1,5 E+1,5 C+0,75 T \\
& E=200+1,2 A_{N}+15 P e+10 N_{R}
\end{aligned}
$$$$
A_{N}=45+4\left(\delta P_{t-1, t}^{t}\right) ; \quad P e=2+4\left(\delta P_{t-1, t}^{t}\right): \quad R=5+3\left(\delta P_{t-1, t}^{t}\right)
$$

mientras que la demanda de chufa por parte de las empresas horchateras responde a:

$$
Q_{x, t}^{d}=-400 P_{x, t}+1,35 P_{x, a, t}+0,75 R+400
$$

A partir de este enunciado, se trata de alterar sucesivamente los supuestos iniciales para calcular equilibrios de mercado suponiendo que se mantienen los precios esperados del momento que se plantó la cosecha; se modifican las expectativas sobre precios; mejora el progreso técnico; se establecen contractualmente los precios a cobrar para el momento en que se recolecte el bien agrario; cambia el precio de un cultivo sustitutivo para el agricultor; se modifica la cantidad de input disponible; la oferta agraria depende de precios desfasados en un periodo; etc.

\section{Competencias específicas y competencias relacionadas con la asignatura}

Con la metodología propuesta tratamos de desarrollar competencias, recogidas tanto en el Proyecto Tuning Educational Structures in Europe, como en los grados en los que se imparten estas asignaturas.

Como es sabido, el concepto de competencia en el EEES se entiende como una combinación dinámica de atributos, en relación a conocimientos, habilidades, actitudes y responsabilidades, que describen los resultados de los aprendizajes de un programa educativo, o lo que los estudiantes son capaces de demostrar al final del proceso educativo
(Proyecto Tunning ${ }^{9}$ ). Estas competencias deben ser entendidas como capacidades y destrezas, en función de los perfiles académicos y de los correspondientes perfiles profesionales (Delgado, 2006).

Por otra parte, en el EEES se distingue entre competencias específicas y competencias transversales. Las competencias específicas son los comportamientos observables que se relacionan directamente con la utilización de conceptos, teorías o habilidades propias de la titulación.

Las competencias específicas a desarrollar con las prácticas planteadas consisten en facilitar la comprensión de los mecanismos de formación de precios en el mercado. Se trata de que, a partir del análisis de las variables que influyen en las funciones de oferta y de demanda de un mercado, los alumnos sean capaces de comprender los conceptos de precio de equilibrio, exceso de oferta y de demanda. También se pretende con esta actividad que los alumnos perciban cómo las alteraciones en las condiciones del mercado, que inducen desplazamientos de la oferta y la demanda, implican la puesta en funcionamiento de mecanismos que permiten la vuelta al equilibrio.

En el caso del mercado agrario, la actividad se centra en analizar la función de oferta agraria, para que el alumno pueda entender qué tipo de factores inciden en las decisiones de los agricultores cuando planifican su producción, y qué factores o variables desvían la producción realizada respecto a la planeada.

En efecto, la oferta agrícola presenta peculiaridades como: desfase temporal entre la toma de decisiones sobre la producción y la llegada al mercado del output agrícola por la dependencia de los ciclos vitales de las plantas y los animales (lo que implica una diferencia entre precio presente y el precio que sirvió a la oferta como referente en periodos anteriores); existencia (a diferencia de la industria) de elementos aleatorios o variaciones no previstas en el output no relacionados con los inputs utilizados porque el sector está sometido a variaciones no previstas y no evitables por los productores (como los climáticos); y la gran importancia del cambio tecnológico en la agricultura (fertilizantes de mayor

${ }^{9}$ Ver González y Wagenaar (Ed.) (2003). 
calidad, biotecnología, nuevas semillas, herbicidas, etc.), lo que provoca que los desplazamientos de la oferta agrícola sean muy importantes.

Así, el objetivo del análisis de la función de oferta es determinar qué tipo de factores afectan e inciden en las decisiones de los agricultores cuando planifican su producción (estudiar la respuesta de la producción planeada ante variables como los precios esperados o precios mínimos garantizados). También se trata de que el alumno comprenda conceptos sobre el funcionamiento del mercado: la curva de oferta de brevísimo plazo (el producto ya ha sido producido y la oferta es prácticamente vertical) o la telaraña de Samuelson.

En la práctica de Economía Mundial/Economía Internacional, tratamos de facilitar a los alumnos la comprensión de cómo las modificaciones en las políticas comerciales afectan a la demanda de importaciones, a la oferta interna y a la oferta mundial (país pequeño versus país grande), lo cual da lugar a variaciones en la producción interna, el consumo y las importaciones.

Además, a partir de las funciones de demanda y de oferta (interna o mundial), el alumno debe ser capaz de determinar quién gana y quién pierde con una cuota o un arancel a la importación: pérdida (ganancia) del consumidor, la ganancia (pérdida) del productor, la renta de la cuota o los ingresos arancelarios, la distorsión en la producción y en el consumo, y la pérdida neta de bienestar. Y, globalmente, puede percibir los efectos que las alteraciones en las barreras al comercio (aranceles y cuotas) pueden tener en el bienestar de una economía. En definitiva, el alumno, puede responder a la pregunta: clas políticas proteccionistas benefician realmente a las economías que los establecen?

Respecto a las competencias transversales, notar que están relacionadas con la formación de un estudiante universitario que se reconocerá en el mundo laboral cuando se deba enfrontar a una situación como profesional o investigador. A su vez, estas competencias transversales pueden ser de tres tipos: instrumentales, interpersonales y sistémicas. Las competencias instrumentales son herramientas para el aprendizaje y la formación (resolución de problemas, técnicas aprendizaje autónomo, análisis y síntesis, organización y planificación, toma de decisiones, habilidades formativas básicas, comunicación oral y escrita, o conocimientos de lenguas extranjeras); las interpersonales son capacidades que permiten mantener una buena relación social (trabajo en equipo de carácter interdisciplinario, razonamiento crítico, compromiso ético, reconocimiento de la diversidad y multiculturalidad, negociación o automotivación); y las sistémicas son aquellas relacionadas con la gestión de la totalidad de la actuación (adaptación a nuevas situaciones, creatividad, liderazgo, iniciativa y espíritu emprendedor, preocupación por la calidad, sensibilidad frente temas medioambientales, gestión de proyectos y gestión por objetivos).

Respecto a las competencias transversales, las prácticas propuestas en este trabajo son prácticas de ordenador, lo que permite desarrollar competencias sobre conocimientos de informática relativos al ámbito de estudio referidas a las habilidades básicas de manejo del ordenador, que se consideran competencias instrumentales. Específicamente podemos con- siderar este tipo de actividades como actividades dirigidas a desarrollar destrezas numéricas y de tecnología informática que permiten resolver problemas numéricos mediante el uso de técnicas basadas en el ordenador o sin él.

Centrémonos en el uso de los programas informáticos. Con el uso de Excel se pretende que los alumnos sean capaces de: introducir fórmulas ${ }^{10}$ de Excel, usar el complemento Solver (por ejemplo, para calcular el equilibrio precio-cantidad en el mercado); usar la función lógica si; usar la función mdeterm (para aplicarla a la resolución de sistemas de ecuaciones mediante la regla de Cramer); e insertar gráficos de dispersión.

Respecto al uso de Maple, TI-Nspire CAS o Mathematica, la resolución de los problemas mediante estos programas debería permitir al alumno familiarizarse con ellos, ya que con este tipo de problemas es posible experimentar muchas aplicaciones del programa. Además, la resolución de los problemas permite al alumno conocer la solución del ejercicio, lo cual debería serle de gran ayuda para a la hora de conformar la hoja de calculo que automatice la solución de cualquier problema estándar.

$\mathrm{Al}$ mismo tiempo, esta actividad supone desarrollar las competencias de resolución de problemas y de capacidad de aplicar el conocimiento (aplicar los conocimientos y la comprensión en la resolución de problemas cualitativos y cuantitativos según modelos previamente desarrollados). Y la forma en la que establezcamos la entrega de la actividad resuelta puede favorecer el desarrollo de otras competencias: podemos ayudar a los estudiantes proporcionándoles un flujo constante de pequeñas tareas y estableciendo como necesario dar las soluciones en fechas fijas señaladas con antelación, con lo que desarrollaríamos la competencia de $c a-$ pacidad de organización y planificación y la de responsabilidad; o podemos establecer que la resolución de problemas debe realizarse en las clases, con lo que podemos desarrollar la competencia de trabajo bajo presión.

Es importante tener presente que las competencias que se definan tienen que ser evaluables, y por ello, la forma en la que se formulen estas competencias debe permitir la identificación de resultados de aprendizaje observables y mensurables. Entraríamos así en el campo de la evaluación de la actividad en la que debemos establecer claramente los criterios a seguir: presentación correcta de las cuestiones planeadas, exactitud en los resultados numéricos, el correcto desplazamiento de las curvas ante cambios en las variables consideradas inicialmente como constates, los razonamientos en los que se basan las soluciones, o el esfuerzo realizado.

\section{Conclusiones}

En el marco del Espacio Europeo de Educación Superior, se considera de especial importancia el desarrollo de competencias profesionales de los alumnos de cara a su inserción profesional, lo que implica que esta nueva orientación hacia estas competencias debe tener un efecto directo sobre la metodología de la enseñanza (y de la evaluación) por parte de los docentes universitarios (Aranda Ogayar et al. 2008). En este sentido, el EEES implica un cambio en la forma en que se imparte docencia en la universidad, lo que exige a los profesores universitarios la innovación y la búsqueda de metodologías activas.

\footnotetext{
${ }^{10}$ Secuencia de valores, referencias de celda, nombres, funciones u operadores de una celda que juntos producen un nuevo valor. Una fórmula siempre comienza por un signo igual $(=)$.
} 
En el caso de la docencia en Economía es necesario diseñar las clases de tal forma que estén presentes las competencias específicas y las transversales, y una forma (no la única) para alcanzar este objetivo puede ser plantearnos el uso de los programas informáticos (Excel y los de cálculo simbólico), para favorecer las competencias instrumentales y favorecer la comprensión de los conceptos y relaciones económicas entre variables.

Esta opción puede tener aspectos positivos. se debe destacar que, con el uso de estos programas, el profesor de economía reduciría su papel de profesor de Matemáticas en el aula, ya que el alumno dispondría de herramientas para realizar unos cálculos matemáticos que debería saber hacer, pero para los que en la práctica encuentra dificultades; $y$ al reducir la repetición de cálculos engorrosos se podría dedicar más tiempo a los contenidos propios de la microeconomía.

Finalmente, no podemos vivir de espaldas a la irrupción de las TIC en el mundo, ni dejar pasar la oportunidad de aprovecharlas para mejorar la enseñanza en el Espacio Europeo de Educación Superior: la introducción de las TIC en la educación no es una nueva moda, es una apuesta por la innovación (Benito y Cruz, 2007).

\section{Bibliografía}

Aranda Ogayar, Manuel; Puentes Poyatos, Raquel; Antequera Solís, José Miguel (2008). Competencias profesionales desde el punto de vista de los empleadores, ex alumnos y alumnos de la Universidad de Jaén. En Emilio José de Castro Silva; José Díaz de Castro (coord.). Universidad, Sociedad y Mercados Globales, Madrid: AEDEM, pp.437-451.

Bengochea Morancho, Aurelia; Camarero Olivas, Mariam; Cantavella Jordá, Manuel; Martínez-Zarzoso, Inmaculada (2002). Economía Internacional. Cuestiones y ejercicios resueltos. Madrid: Prentice Hall.

Benito, Águeda; Cruz, Ana (2007). Nuevas claves para la Docencia Universitaria en el Espacio Europeo de Educación Superior. Madrid: Narcea.

Blanco, Juan Manuel; Aznar, Juana (2004). Introducción a la Economía. Teoría y práctica. Madrid: McGrawHill.
Cahill, Miles; Kosicki, George (2000). Exploring economic models using excel.Southern Economic Journal, vol. 66, no. 3 (January), pp. 770-792.

Cahill, Miles; Kosicki, George (2001). A Framework for Developing Spreadsheet Applications in Economics. Social Science Computer Review, Vol. 19, 2, pp.186-200.

Delgado García, Ana María (Coord.) (2006). Evaluación de las competencias en el Espacio Europeo de Educación Superior. Barcelona: J.M. Bosch Editor.

González, Julia; Wagenaar, Robert (Ed.) (2003): Tuning Educational Structures in Europe. Bilbao: Universidad de Deusto.

http://www.relint.deusto.es/TUNINGProject/spanish/doc fase1/Tuning\%20Educational.pdf

Katz, Arnold; Becker, William E. (1999). Technology and the Teaching of Economics to Undergraduates. Journal of Economic Education, 30(3), pp.194-99.

Mixon, J. Wilson; Tohamy, Soumaya (2000). Using Microsoft Excel in Principles of Economics, Computers in Higher Education Economics Review (CHEER), Vol. 14, Issue 2.

Mixon, J. Wilson (2005): Analyzing Subsidies in Microsoft Excel, Journal of Economic Education, Vol. 36, 2.

Murphy, Barry (1995a): Examples for Economists with DERIVE 3.0: "Long- and Short-run Costs", Computers in Higher Education Economics Review (CHEER), Volume 9, Issue 2.

Murphy, Barry (1995b): Examples for Economists with DERIVET ${ }^{\mathrm{TM}}$ : General Equilibrium for a Cobb-Douglas Exchange Economy, Computers in Higher Education Economics Review (CHEER), Volume 9, Issue 3.

Tohamy, Soumaya; Mixon, J. Wilson (2000): Using Microsoft Excel to illustrate gains from trade", Business Quest http://www.westga.edu/ bquest/200o/excel.html.

Tohamy, Soumaya; Mixon, J. Wilson (2002). Comparing Trade Instruments Using Spreadsheets, Social Science Computer Review, 20, pp.187-193.

Whigham, D. y Whyte, J. (1999) A graphic view of input output relations with Excel, Computers in Higher Education Economics Review, vol. 13, 1. 